\title{
Communication as a Medical Procedure
}

\section{Werner Bauer}

Dr méd., ancien président de l'Institut suisse pour la formation médicale postgraduée et continue (ISFM)

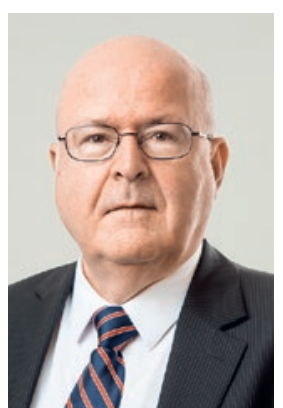

La communication fait partie des objectifs généraux de la formation postgraduée, au même titre que la prise de décision éthique, la conduite d'équipe, l'économie de la santé et la gestion des erreurs. Si l'importance de chacun de ces objectifs est incontestée, leur transmission peut s'avérer compliquée sur le terrain, que ce soit à l'hôpital ou au cabinet. Elle est souvent reléguée au second plan.

Il y a quelques années, l'Institut suisse pour la formation médicale postgraduée et continue (ISFM) a mené une enquête auprès des médecins venant d'obtenir leur titre de spécialiste [1]. La grande majorité des plus de 1000 personnes ayant répondu au sondage se sont déclarées satisfaites de la formation postgraduée dans leur domaine de spécialisation et ont confirmé qu'elle leur a permis d'acquérir les compétences nécessaires pour leur activité. Mais elles ont aussi été nombreuses à signaler certaines lacunes, précisément dans les objectifs de formation généraux: conduite d'entretien, évaluation de la capacité de travail, économie de la santé et analyse des erreurs (dans cet ordre). Fait intéressant, ces lacunes ont été relevées dans toutes les disciplines, à des degrés divers; un manque de formation en conduite d'entretien a même été signalé par les psychiatres fraîchement diplômés.

Suite à cette enquête, nous avons demandé à plus de 500 médecins-cheffes et chefs s'ils avaient constaté des lacunes chez leurs internes et s'ils avaient besoin d'aide pour l'enseignement des objectifs de formation problématiques. Les lacunes soulevées étaient pratiquement les mêmes que celles de la première enquête avec, en tête de liste, la communication, la gestion de conflits, le leadership et l'économie de la santé. Les lacunes étant désormais connues, il s'agit à présent de les combler. Intéressons-nous de plus près à un objectif éminemment important: la communication. Selon de nombreuses études, «on ne peut pas ne pas communiquer». C'est peut-être vrai en général, mais je dois néanmoins m'inscrire en faux: oh que si, il est tout à fait possible de ne pas communiquer! Pour la patientèle, c'est par exemple le cas lorsqu'un entretien est mené avec maladresse, sans grande conviction ou à la va-vite, la laissant sans réponse, voire lui donnant l'impression de ne pas être prise au sérieux. Cela arrive. Pas toujours de manière aussi flagrante et rarement intentionnellement, mais non sans conséquence. Nous connaissons malheureusement toutes et tous des exemples.

Un article paru dans les Annals of Internal Medicine a donc tout particulièrement retenu mon attention: Time out before talking: Communication as a medical procedure [1]. L’idée qu'il développe est aussi simple que convaincante: de l'investigation clinique à l'opération, toutes les mesures ou interventions médicales reposent sur des connaissances et compétences qui doivent être acquises.

Pourquoi alors ne pas faire de même avec la conduite d'entretien en lui conférant le statut de procedure comme n'importe quelle autre intervention médicale? Concrètement, cela voudrait dire que pour mener un entretien, il faudrait d'abord acquérir certaines compétences. Cela impliquerait aussi que, à l'instar d'une ponction lombaire ou d'une échographie, la communication devrait faire l'objet d'une préparation plus ou moins longue afin d'éviter qu'elle ne se réduise à quelques mots prononcés entre le lit et la porte ou à de brefs regards lancés par-dessus l'écran. Enfin, cela signifierait que, comme pour toutes les autres mesures et interventions médicales, la communication peut avoir des complications et des effets indésirables, par exemple sous la forme de réactions inattendues à plus ou moins long terme.

Les auteurs concluent: "Describing communication as a procedure does not dictate rigidly scripted or stereotyped practice. Skilled communication requires nuance, adjustment, and careful thought in complex interpersonal interactions. Just as surgery is a technical intervention and a practiced art form, communication procedures require both thoughtful structure and flexible skill.»

L'expérience montre que la communication n'est pas simplement quelque chose d'inné - nous ne faisons pas toutes et tous naturellement preuve de la même habileté lorsqu'il s'agit de communiquer. Au contraire, la communication est quelque chose que l'on peut en grande partie apprendre et travailler. Pour cela, je recommande vivement la brochure "La communication dans la médecine au quotidien" de l'Académie suisse des sciences médicales, disponible en ligne et en version imprimée [2]. 